\title{
La influencia del precio y las estrategias de comunicación visual basadas en simbología cultural sobre la preferencia de marcas ecológicas y consumo sostenible
}

Haidy Johana Moreno C.

The influence of price and the strategies

of visual communication based on cultural

symbols of ecological brands and

sustainable consumption preferences

L'influence des prix et des

stratégies de communication visuelle dans le

symbolisme culture/ sur la préférence de

consommation de marques

respectueuses de l'environnement et apportant

leur soutien au commerce équitable

Influência do preçu e as

stratégias de comunicaçãa visual baseadas

em simbolagia cultural sabre a preferência de

marcas eculógicas e consumo sustentável

* Mestría en Psicología del Consumidor.

Docente Programa de Mercadeo,

Facultad de Estudios en Ambientes Virtuales.

Universidad EAN. 


\section{RESUMEN}

Esta investigación pretende establecer la influencia del precioy las estrategias de comunicación visual, basadas en simbología cultural sobre la preferencia de marcas ecológicas en la categoría de productos de aseo en la ciudad de Bogotá, buscando fomentar el consumo sostenible en los hogares. Para ello, se realizaron dos fases: un estudio de tipo descriptivo para establecer la estrategia de precio más persuasiva para el consumidor en un producto ecológico en la categoría de aseo. Posteriormente en la fase dos, se realizó un estudio experimental mediante un diseño factorial $2 \times 2 \times 2$, donde la primera variable independiente fue comunicación visual, la segunda, el enmarcamiento ecológico y la tercera, el precio; variable que actuó como elemento de influencia utilizada en cada grupo experimental. Los participantes fueron expuestos a estímulos visuales de tipo bidimensional y tridimensional, relacionados con las estrategias de precio; los resultados muestran, que de la manera como se comunica el precio, se presenta una alta incidencia en la preferencia de compra, debido a que los consumidores son más perceptibles a los descuentos y a las ofertas. Otro de los hallazgos relevantes fue la estrategia de posicionamiento en marcas verdes, las cuales deben generar en los consumidores una garantía que realmente es un producto que aporta al cuidado medioambiental y para ello, se requiere de información precisa y detallada en el diseño, los empaques, los envases, la marca y demás atributos del producto; pues en definitiva, en términos publicitarios que la comunicación visual en consumo sustentable cumple una función también informativa.

\section{ABSTRACT}

This paper aims at describing the influence of the price and strategies of visual communication based on cultural symbols of ecological brands preferences in the category of cleaning products in the city of Bogota, trying to increase the sustainable consumption of energy at home. For this reason, two steps were followed: a descriptive study to determine the most persuasive price strategy for consumers for an ecological cleaning product.

Subsequently, in phase two, a pilot study was conducted using a $2 \times 2 \times 2$ factorial design, where the first independent variable was visual communication; the second one was the ecological framing and third one, the price; variable that acted as an element of influence used in each experimental group. The participants were exposed to visual two-dimensional and three-dimensional stimuli, related to pricing strategies. The results show that the way the price is communicated has a high incidence in purchasing preference because consumers are more perceptible to discounts and offers. Another relevant finding was the positioning strategy in green brands, which should generate in consumers a guarantee that it really is a product that provides the environmental care and therefore requires accurate and detailed information on the design, packaging, branding and other product attributes, seeing that ultimately and in advertising terms, visual communication in sustainable consumption also plays an information role.

\section{Palabras clave}

Influencia social Comunicación visual

Marketing ecológico Marcas verdes

Comportamiento sostenible

\section{Keywords}

Social influence

Visual communication

Ecological marketing

Green brands

Sustainable behaviour 


\section{RESUMÉÉ}

Cette recherche vise à établir l'influence des prix et des stratégies de communication visuelle basés sur le symbolisme culturel et sur la préférence accordée aux marques respectueuses de l'environnement. Nous nous pencherons principalement sur les marques de produits ménagers en vente dans la ville de Bogotá qui recherchent à promouvoir une consommation des ménages respectueuse de l'environnement. Pour mener à bien cette investigation, nous avons réalisé une étude descriptive de la stratégie tarifaire la plus persuasive pour le consommateur d'un produit biologique dans la catégorie des produits ménagers.

\section{RESUMO}

Esta investigação pretende estabelecer a influência do preço e as estratégias de comunicação visual, baseadas em simbologia cultural, sobre a preferência de marcas ecológicas na categoria de produtos de limpeza na cidade de Bogotá, tentando fomentar o consumo sustentável nos lares. Para isto, realizaram-se duas fases: um estudo de tipo descritivo para estabelecer a estratégia de preço mais persuasiva para o consumidor para um produto ecológico na categoria de limpeza.

\section{Mots clefs}

Prix

Influence sociale

Communication visuelle

Marketing écologique Attitudes

Marques vertes

Commerce équitable

Préférence, éco-

consommation

\section{Palavras-chave}

Preço

Influência social

Comunicação visual

Marketing ecológico

Atitudes

Marcas verdes

Comportamento sustentável Preferência

Consumo ecológico 


\section{INTRODUCCIÓN}

E n los últimos años, el mundo ha sido el escenario de los efectos nocivos del crecimiento económico sobre el medio ambiente, en consecuencia, cada día más personas apoyan la implementación de un modelo global de desarrollo sustentable, concepto que fue instaurado a partir del informe realizado por la Comisión Mundial sobre Ambiente y Desarrollo en el año de 1987, entendiéndose como "aquel desarrollo que satisface las necesidades de las generaciones presentes, sin comprometer la capacidad de las generaciones futuras para satisfacer sus propias necesidades".

Castellanos (2009) afirma que el desarrollo sustentable combina tres criterios: el económico, el social y el ambiental. El ambiental es considerado como el más importante, pues incorpora la idea de equidad intergeneracional en el consumo de recursos naturales y es cada vez más evidente para los consumidores. Existen diversos estudios que exponen el creciente interés en los consumidores frente al deterioro medioambiental, tal como lo menciona Fraj y Martínez (2003), quienes observaron en las últimas décadas la preocupación de los consumidores por la amenaza medioambiental y cómo esta se refleja en su comportamiento de compra, en la que influyen los principios medioambientales, cuando adquieren un producto o un servicio o en el cambio de sus hábitos de consumo cuando dejan de comprar determinados productos por sus efectos contaminantes, así también lo concluye el estudio desarrollado por Sánchez et al. (2001), que explica los diferentes segmentos del mercado y la disposición a pagar más por un producto o alimento ecológico.

En consecuencia el presente artículo, pretende caracterizar la eficacia de las estrategias de comunicación visual basadas en símbolos culturales para productos ecológicos cuando son expuestas en comparación con variables de naturaleza económica, en este caso el precio, lo cual denota un significativo aporte para la investigación en consumo sustentable, ya que además de explicar el comportamiento del consumidor, se acerca más a la realidad social de consumo, tal como lo plantea
Quintanilla (2010), cuando se refiere a la forma en la que la economía influye sobre las conductas, pensamientos, actitudes, motivaciones, emociones y expectativas de los ciudadanos y, recíprocamente, todas ellas influyen sobre la economía. Por esta razón, la economía y la conducta social reproducen un proceso interactivo y retroalimentado que influye en la decisión de compra. Por ende la dinámica entre la comunicación y el precio, para efectos del presente estudio, buscan promover la preferencia hacia productos ecológicos que fortalezcan en los consumidores un estilo de vida sostenible.

Para alcanzar dicho propósito, el presente artículo, inicia con una revisión de las tendencias en consumo sustentable, lo que posteriormente condujo a una investigación de tipo exploratorio sobre la categoría de productos de aseo en grandes superficies y almacenes de cadena en la ciudad de Bogotá, buscando establecer así los diferentes precios del mercado para estos productos.

Posteriormente, se revisó la investigación realizada por Calderón (2013) en donde se contemplan los aspectos relevantes en la comunicación visual para las marcas ecológicas, los cuales se validaron en sesiones grupales con amas de casa de estratos dos al cinco, en donde las participantes fueron expuestas a diferentes productos de la categoría de aseo, incluyendo las marcas no ecológicas creadas por Calderón (2013). Se concluyó que cierto perfil de consumidor está dispuesto a pagar un poco más por adquirir un producto siempre y cuando este garantice el cuidado del medio ambiente, señalando así una brecha de conocimiento en el estado del arte actual sobre el consumo sustentable. Consecutivamente se complementó con la revisión de los supuestos teóricos de la influencia social y las estrategias de comunicación visual en marcas verdes, donde la percepción del precio toma protagonismo a lo largo de la etapa experimental, como variable nominal que permite identificar el grado de preferencia en los participantes, determinando así diferentes conclusiones que responden tanto a las hipótesis planteadas como a los objetivos propuestos en el estudio. 


\section{PRINCIPALES REFERENTES TEÓRICOS}

\subsection{La educación para el consumo sustentable: el verdadero aprendizaje del consumidor}

Una de las estrategias de promoción hacia el consumo sustentable, es la educación y el aprendizaje, puesto que es allí donde se comprenden críticamente los problemas ambientales y su relación con el contexto social, la comunidad y la globalización. Terrón (2009), propone que: "la educación ambiental emerge de la idea de que la educación es un medio para el cambio social, sus sujetos centrales son las nuevas generaciones que han de dar luz a ese cambio; su finalidad es promover una transformación social que se manifieste en valores, actitudes y relaciones para vivir en armonía con la naturaleza y mejorar la calidad de vida humana". De esta forma, la educación para el consumo sustentable, permite la construcción de una cultura ambiental que se desarrolla desde el aprendizaje en los primeros años de la escuela, hasta llegar al uso responsable y a la adquisición de bienes y servicios en edad adulta.

Esta situación involucra al sector empresarial, siendo el encargado de implementar, en un primer lugar, procesos productivos responsables con el medio ambiente a través de programas de responsabilidad social empresarial, y en segundo lugar, en el desarrollo y la innovación de productos ecológicos, los cuales, mediante acciones del Marketing verde y la publicidad, ofrecen una alternativa de compra para ciertos segmentos de mercado que muestran mayor preocupación por el deterioro medioambiental.

Lo expuesto anteriormente, se fundamenta principalmente en la construcción de las representaciones sociales, puesto que la educación para el consumo sustentable, tal como lo afirma Jiménez (citado por Terrón, 2009), es una transmisión y socialización en lo que compete a sus contenidos y forma. Sólo mediante el acto educativo, los individuos son motivados por determinadas formas de pensar (creencias básicas dominantes de una época) que a su vez expresan una determinada forma de pensar, vivir en el mundo y en la manera de relacionarse. Entonces, el aprendizaje se adapta a los estilos de vida actuales, siendo más acorde con la realidad.

Por tal razón, las empresas y organizaciones, deben trabajar más en función de la educación para el consumo, más allá de intentar alcanzar un posicionamiento o una participación en el mercado, buscando alcanzar estándares que permitan que un producto verde cumpla con las características correctas, logrando que mediante su uso, se garantice la sostenibilidad de las generaciones futuras, y no estrictamente como desde una mirada empresarial. Por lo tanto, la comunicación visual, desde el punto de vista empresarial, ayudará a incrementar la conciencia social, para lo cual la publicidad, los símbolos y el empaque de productos ecológicos, más allá de ser una imagen para vender, debe realmente transmitir la protección medioambiental y el mejoramiento de la calidad de vida de los individuos y de las comunidades.

\subsection{Representaciones sociales y comunicación visual para marcas verdes. Construcción de la imagen visual desde la simbología cultural}

La imagen visual es fundamental en la comunicación de productos ecológicos, pues permite que se lleven a cabo las asociaciones correspondientes. Por lo tanto, si un consumidor percibe ciertos símbolos en la publicidad de una marca verde, este deberá comunicar las características amigables con el medio ambiente de dicho producto o servicio (Velandia 2012). Sin embargo, el consumidor no siempre hará estas asociaciones y es muy posible que tampoco las interprete como un hecho que contribuye a preservar los recursos naturales. 
Es claro entonces, que si un artículo ecológico muestra un significado, al mismo tiempo nos estará comunicando un propósito, el cual deberá ser percibido por el consumidor en el menor tiempo posible. Para ello, la comprensión de la dimensión simbólica del consumo ambiental, desde la teoría de las prácticas y representaciones sociales de Moscovici $(1961,1976)$, se viene posicionando como una valiosa fuente de investigación para la psicología social, gracias al interés de querer interpretar los procesos cognitivos y simbólicos de las personas y su comportamiento frente al ambiente natural.

\subsection{Estrategias de posicionamiento en marcas verdes ¿Cómo posicionar una marca ecológica?}

En la actualidad, gran número de marcas se posicionan por sus beneficios emocionales (Aaker, 2000). Dicha tendencia aplica también como estrategia en el posicionamiento verde, que según lo plantea Hartmann et al. (2002), puede basarse en tres tipos diferenciados de beneficios emocionales: la sensación agradable de aportar a la sociedad (Ritov y Kahnemann, 1997), en beneficios de autoexpresión a través del consumo socialp.212 mente visible de marcas ecológicas (consumo simbólico) (Belz y Dyllik, citado por Hartmann et al., 2002) y en experiencias emocionales relacionadas con la naturaleza.

En este último caso, se busca transformar la imagen en un medio que permita evocar sensaciones como las experimentadas en el contacto con la naturaleza, tal como lo mencionan, Kals, Schumacher y Montada (1999), cuando afirman que: "el posicionamiento verde se basa en una sensación de afinidad emocional hacia la naturaleza, como por ejemplo, el amor a la naturaleza o el sentimiento de integración en la naturaleza". En efecto, estas emociones se traducen en sentimientos como: el experimentar una sensación de felicidad o alegría en el contacto con el entorno, lo que en definitiva definen los paradigmas en la formulación de estrategias de comunicación visual para productos ecológicos.
Es por ello, que los anunciantes deben buscar mayor claridad en la comunicación de los atributos diferenciadores de las marcas verdes, pues es el consumidor quien valora los beneficios, evalúa el precio y decide la compra. De este modo, el precio como variable del mercadeo, ofrece el equilibro perfecto entre los atributos del producto y el desembolso monetario que realiza el consumidor, por ello, hablamos del precio como herramienta de comunicación de valor y variable de gran influencia sobre la preferencia y adquisición final de una marca o producto.

\subsection{El precio y la preferencia ¿Cuál es el precio que un consumidor estaría dispuesto a pagar?}

Los procesos de percepción de precio son de gran interés para la comprensión de las decisiones de compra en el consumidor, tal como lo mencionan Lichtenstein, Ridgway y Netemeyer (1993): "La forma en que los consumidores perciban e interpreten las estructuras de precios presentes en un mercado determinará en gran medida el proceso de búsqueda y evaluación de la información relativa a los productos y servicios durante un episodio de elección". Dicho planteamiento se fundamenta en diferentes enfoques teóricos, siendo dos los más relevantes; el primero de ellos, la teoría de EstímuloOrganismo-Respuesta de Jacoby y Olson (1977), cuyo planteamiento es que los precios de venta al público constituyen un estímulo o señal que activa el proceso de percepción de los consumidores. La parte correspondiente al organismo refleja el proceso psicológico de la señal precio, adquisición de información, codificación y almacenamiento de la misma, como resultado la respuesta se reflejará en el comportamiento de compra o no compra de los consumidores.

Ensegunda instancia, seencuentra la interpretación de la Teoría de los precios psicológicos, que permite evidenciar que el precio no es sólo un número, puesto que estudios como los de Monroe (1992) y Oubiuña (1997), mencionan la influencia de las terminaciones de los precios sobre la percepción de los mismos. Lo 
que evidencia que estos influyen directamente sobre el proceso cognitivo de los consumidores y son un elemento de comunicación e interacción durante el proceso de toma de decisiones, siendo una valiosa fuente de información y de comparación para realizar la compra. Por tal razón, desde la investigación del consumidor y la gestión de Marketing, resulta interesante conocer cuáles son aquellos factores que determinan el tipo de precio de referencia ya sea interno o externo que utilizan los consumidores en sus evaluaciones de compra.

Durante el proceso de compra, el precio observado, bien sea a manera de oferta, descuento o promoción, logrará provocar en el consumidor resultados inmediatos, sin embargo, existe en la percepción de precios del consumidor otras categorías que desde luego no producen efectos tan inmediatos, por el contrario, pueden generar un efecto de estimación frente al precio que se comunica, se trata de los precios de referencia, aquellos que permite que el consumidor lleve a cabo las evaluaciones pertinentes para la decisión final de compra.
Por ello la gestión de mercadeo y comunicación publicitaria, buscan una mejor claridad perceptiva, mediante la transmisión de imágenes, colores llamativos, términos promocionales, descuentos, ofertas, entre otras acciones estratégicas del precio, con las que se proyecta atraer mayor número de clientes potenciales; para ello, una alternativa ideal son los precios psicológicos, considerando que uno de los factores críticos de la implementación de las estrategias de precios, es la forma en que los consumidores perciben los precios de los productos (Castro y Rosemberg, 2000) y de qué manera el consumidor como ser racional que procesa información, la utiliza para construir la preferencia hacia una marca o producto. Así, la idea es formar con la intención de realizar o no una conducta de compra específica, tal como lo expone la teoría de la acción razonada de Ajzen y Fishbein (1980), que demuestra que la

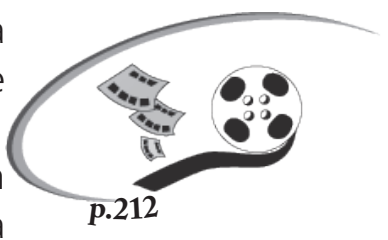
mayoría de los comportamientos sociales relevantes están bajo el control del sujeto, por tanto revela su capacidad decisiva que en últimas determinará la actitud hacia la compra es decir la evaluación de los atributos y los beneficios, factor determinante al momento de adquirir un producto ecológico. 


\section{METODOLOGÍA}

Para el desarrollo del estudio se realizó un experimento de tipo factorial que se llevó a cabo en dos fases: la primera de tipo descriptivo, cuyo propósito es el de especificar las propiedades, características, rasgos y tendencias importantes de cualquier fenómeno, grupo o población. Y la segunda fase de tipo experimental, buscando establecer el posible efecto de los precios y los tipos de comunicación visual basados en simbología cultural sobre la preferencia de marcas verdes en la categoría de aseo.

\subsection{Muestreo y participantes}

Para la fase 1, se realizó una selección de los participantes tomando en consideración características representativas de las amas de casa, quienes toman decisiones de compra sobre los productos de la categoría de aseo, se toman además variables de tipo demográfico, tales como: edad, sexo, estrato. Esta última, caracterizó la sesión grupal en la cual participaron las amas de casa. Se realizó una sesión para los estratos dos y tres y otra para cuatro y cinco.

Vale la pena mencionar que en la primera fase, se toman únicamente mujeres, debido a que no requieren de mayor información con relación a la categoría de productos de aseo, puesto que en la mayoría de los casos, conocen ampliamente las marcas, las presentaciones y los atributos que hacen diferenciadora cada marca, así como los precios, ya que están constantemente en contacto con este tipo de productos, tal como lo menciona McGuire (Citado por Briñol y otros), "las mujeres poseerían una mayor capacidad para comprender y recibir los mensajes y, por tanto, para ser persuadidas por la posición y beneficios defendidos en ellos".

Posteriormente, en la fase experimental, la muestra se calcula con el apoyo del Software Piface, versión 1.72, realizándose una distribución aleatoria de sujetos en los diferentes grupos experimentales. A lo largo de la etapa experimental, se seleccionaron en total 80 participantes correspondientes al perfil manifiesto en la fase 1, lo que implica un grupo de 10 individuos, por condición experimental, arrojando

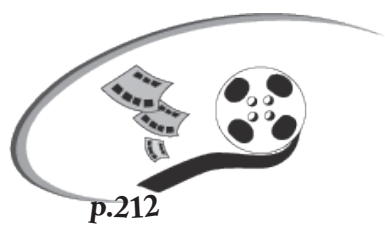
como resultado un error tipo 1 menor al $0.05 \%$ y una potencia superior a 0.8 , compuesta por hombres y mujeres que toman decisiones de compra en el hogar.

\subsection{Variables}

Se establecen variables independientes, como el precio, la comunicación visual basada en simbología cultural y el tipo de enmarcamiento bidimensional y tridimensional en escalas de medición nominal. Por último, la variable preferencia se presenta en una escala de medición continua.

\subsection{Procedimiento}

Durante la fase 1, a las participantes convocadas se les aplicó un filtro para establecer, si en efecto toman decisiones de compra en el hogar, marcas de la categoría y promedios de compra mensuales, para determinar cuál es la estrategia más persuasiva de precio para un producto ecológico de la categoría de aseo, lo cual se evaluó, en dos momentos: la primera a través de una actividad lúdica en la que se exhibieron las principales marcas de la categoría, en donde los participantes estimaron el precio de cada una de las marcas expuestas, lo que se explica desde la teoría de los precios de referencia, que son definidos como: aquel precio interno (recuerdo de la última compra realizada), un precio esperado o alguna creencia sobre el precio de un producto en el mismo mercado (Castro y Rosemberg 2000), logrando establecer además, cuál es la percepción de precio para una marca verde. 
Finalmente la segunda etapa de la sesión grupal, se desarrolló mediante la aplicación del instrumento: medición de la persuasión de la estrategia de comunicación del precio. En donde las participantes evauaron, cuál de las estrategias de precios (Castro y Rosemberg, 2000) es más atractiva para las amas de casa participantes, las estrategias de precio a evaluar fueron: precio de descuento: más cantidad por el mismo precio, pague 1 lleve 2, igual cantidad por menor precio; precio impar: terminado en 9; precio de prestigio: por encima de la competencia; precio de penetración: por debajo de la competencia; precio con asterisco: precio base más IVA; precio mínimo: cuánto vale cada gramo o litro del contenido.

Posteriormente, para la fase 2 experimental, los participantes fueron ubicados aleatoriamente, luego aplicar un filtro de reclutamiento en donde se garantizaba en primer lugar, que no utilizarían productos ecológicos, orgánicos y biodegradables en la categoría de aseo. Los ocho grupos experimentales, fueron expuestos a cada una de las estrategias de comunicación visual de tipo bidimensional y tridimensional, exhibidas con la variable precio: terminado en número impar y de oferta, los cuales fueron seleccionados como resultado del análisis de la fase 1. Se distribuyeron de manera equitativa las variables nivel socio-económico y sexo, que corresponden al perfil socio demográfico, vale la pena aclarar que cada individuo, fue expuesto únicamente a un estímulo y participó en un solo grupo experimental.

\subsection{Análisis de datos}

El análisis de los datos en la fase 1 descriptiva, se realizó a través de codificación abierta (conceptualización de categorías principales) y codificación axial (relaciones entre categorías), determinando así las codificación selectivas (conceptos principales), con el apoyo del software Atlas. Ti. Versión 7. 3, identificando, los principales hábitos y rutinas del hogar a la hora de la limpieza, junto con las marcas más representativas, se identifican además los significados asociados a las marcas verdes. Para los datos obtenidos en la fase 2 el análisis de datos, se llevaron a cabo con ayuda del Software IBM SPSS 21, mediante un modelo de análisis factorial bajo ANOVA de tres vías, utilizando las herramientas estadísticas del Modelo lineal general univariante, en el cual la variable dependiente es la preferencia y tres variables independientes: tipo de enmarcamiento: ecológico y no ecológico, tipo de comunicación, bidimensional y tridimensional y como última variable el precio uno terminado en número impar y el precio dos de oferta.

\subsection{Resultados}

Los resultados del modelo factorial de varianza para el análisis de datos de la presente investigación, evalúan el efecto individual de una variable sobre el conjunto de los tres factores: tipo de enmarcamiento, tipo de estrategia de comunicación visual y tipo de precio. Para este caso, se pretende analizar un efecto de interacción entre los tres factores (variable independiente) y el efecto sobre la preferencia (variable dependiente).

En este tipo de análisis factorial, existe una hipótesis nula por cada factor, definiendo así:

La hipótesis nula referida a un factor, afirma que las medias poblacionales definidas por los niveles del factor son iguales.

Las hipótesis preferidas al efecto de una interacción afirman que tal efecto es nulo. A continuación, se observan los datos obtenidos mediante este tipo de análisis bajo ANOVA de tres vías. 
Tal como se fundamenta el modelo de análisis factorial de varianzas bajo ANOVA de tres vías, se plantea una hipótesis nula por cada factor, por lo tanto, para los resultados anteriores se expone lo siguiente:

Ho nula: el promedio de preferencia para todos los niveles del factor precio son iguales.

Ho alternativa: el promedio de preferencia es diferente para algún nivel del factor precio.
De acuerdo con los resultados, obtenidos en la prueba de medias del análisis de varianzas, los efectos inter sujetos muestran que se encontraron efectos principales entre la variable dependiente preferencia y la variable independiente precio, con un valor de $\mathrm{P}$ 0.043 (Tabla 1), lo que nos permite concluir que los promedios de las preferencias son distintos para el factor precio, en este caso el precio impar y precio con oferta. Revisaremos a continuación algunos estadísticos descriptivos que nos permitan comprender esta relación:

Tabla 1. Resultados de pruebas de efectos intersujetos variable dependiente: preferencia. Variable independiente: estrategia de comunicación del precio.

\begin{tabular}{|l|l|l|l|l|l|}
\hline \multicolumn{7}{|c|}{ Variable dependiente: PREFERNCIA } \\
\hline & $\begin{array}{c}\text { Tipo III de } \\
\text { suma de } \\
\text { cuadrados }\end{array}$ & gl & $\begin{array}{c}\text { Cuadrático } \\
\text { promedio }\end{array}$ & \multicolumn{1}{c|}{ F } & Sig. \\
\hline Modelo corregido & $25,971 \mathrm{a}$ & 7 & 3,710 & 2,211 &, 043 \\
\hline Interceptación & 860,508 & 1 & 860,508 & 512,782 &, 000 \\
\hline PRECIO & 10,823 & 1 & 10,823 & 6,449 &, 013 \\
\hline TIPOCOMU & 4,152 & 1 & 4,152 & 2,474 &, 120 \\
\hline TIPOENMAR &, 160 & 1 &, 160 &, 095 &, 759 \\
\hline $\begin{array}{l}\text { PRECIO * } \\
\text { TIPOCOMU }\end{array}$ & 2,406 & 1 & 2,406 & 1,434 &, 235 \\
\hline $\begin{array}{l}\text { PRECIO * } \\
\text { TIPOENMAR }\end{array}$ & 3,170 & 1 & 3,170 & 1,889 &, 174 \\
\hline $\begin{array}{l}\text { TIPOCOMU * } \\
\text { TIPOENMAR }\end{array}$ & 5,113 & 1 & 5,113 & 3,047 &, 085 \\
\hline $\begin{array}{l}\text { PRECIO * } \\
\text { TIPOCOMU * }\end{array}$ &, 147 & 1 &, 147 &, 087 &, 768 \\
\hline TIPOENMAR & 146,795 & 79 & & & \\
\hline Error & 120,824 & 72 & 1,678 & & \\
\hline Total & 1007,303 & 80 & & & \\
\hline Total corregido & 146,795 & & & \\
\hline
\end{tabular}

Fuente. Elaboración propia de la autora. 
Tabla 2. Estadísticos descriptivos de la estrategia de comunicación del precio

\begin{tabular}{|l|l|l|l|}
\hline \multicolumn{4}{|c|}{ Variable dependiente: Prepre } \\
\hline Precio & Media & $\begin{array}{l}\text { Desviación } \\
\text { estándar }\end{array}$ & $\mathbf{N}$ \\
\hline 1,00 & 2,9119 & & \\
\hline 1,13729 & 40 & & \\
\hline 2,00 & 3,6475 & 1,48089 & 40 \\
\hline Total & 3,2797 & 1,36315 & 80 \\
\hline & & & \\
\hline
\end{tabular}

Fuente. Elaboración propia de la autora.
De acuerdo con la revisión de las medias de los datos expuestos se evidencia que para el precio 2, (precio con oferta) la media es de 3.64, superior a la media de la estrategia del precio 1 (terminado en número impar) que fue de $\tan$ solo 2.91, lo que indica que el factor precio para todos los niveles no es igual, siendo entonces el precio 2 el que mayor influye en la preferencia, que como tal está orientada a responder a la intención de compra en cuanto a: atractividad del precio (persuasión), alcance del precio (poder adquisitivo), coherencia del precio y atributos (percepción de valor) y disposición a pagar más por el producto (precios de referencia), elementos que fueron abordados teóricamente en el apartado de precios.

\section{CONCLUSIONES}

$\mathbf{L}$ os resultados de la fase 1, arrojan que los precios con terminación en número impar o los precios por debajo de un número redondo tales como: $99,98,97$, son más llamativos para el consumidor, al igual que los comunicados con descuento u oferta, también resultan ser muy atractivos. Por su parte, los precios impares son una estrategia que fijan precios terminados en números impares, por ejemplo, un producto con precios como US $\$ 9,99$ o US $\$ 9,95$ en lugar de US $\$ 10$. Aunque para ambos casos los precios son prácticamente los mismos, los consumidores tienden a asociar los números impares con descuentos, así que en la mayoría de los casos, el artículo con un precio impar, será más atrayente para un cliente que posiblemente busca oferta o un precio económico. Así como lo expone California State University, puesto que las ofertas y los descuentos psicológicos son una estrategia que permite comparar un precio con rangos de precio concreto, para simular el efecto de un descuento, aunque en muchos de los casos, el precio nunca se redujo.

En este orden de ideas Baños (2012), plantea que las estrategias de precios basadas en la psicología, reconocen que los consumidores no siempre actúan racionalmente hacia los precios, ya que en muchos de los casos, la gente no elegirá automáticamente el precio más bajo, sino que reaccionará emocionalmente a diferentes estrategias. Baños (2012), también propone que las estrategias psicológicas, intentan evocar reacciones afectivas, un ejemplo de ello lo demuestran Thomas y Morwitz (2008), quienes investigaron, que una diferencia mínima en la presentación de los precios puede tener consecuencias positivas en las ventas; los resultados de este estudio demuestran que el primer dígito del precio rebajado o en oferta, más que los dos últimos, es responsable de la diferencia percibida por el comprador, diferencia que termina siendo importante en la elección del producto promocionado.

El estudio también menciona que el efecto se produce al cambiar, por ejemplo, de 3,00 a 2,99 ya que el céntimo o centavo rebajado hace que el primer número cambie de tres a dos, por ende, la influencia psicológica es mayor en el llamado efecto nueve, razón por la cual son atractivos los precios terminados en número impar, y son muy prácticos a la hora de implementar una estrategia comercial. 
En consecuencia, el análisis de los datos en donde se encontraron efectos principales en la condición conformada por la variable dependiente preferencia y la de precio, nos permitió concluir, que los promedios de las preferencias son distintos para el factor precio, y para este caso, específicamente el precio con oferta lo que se puede relacionar directamente con la propuesta de Coulter \& Coulter (2007), donde se menciona que lo más común es que se ofrezca un precio de venta más bajo y permite que se compare con otro al que probablemente el consumidor lo denomina regular, teniendo en cuenta que el precio regular (precio sin descuento) funciona como un marco de referencia externo que hace que los consumidores perciban un beneficio reducido de la oferta promocionada.

El asociar un menor sacrificio con el descuento ofrecido, consecuentemente, llevará a que la comparación de precios genere percepciones de valor más favorables hacia el consumidor. En la gestión de mercadeo, son muy utilizadas estas actividades promocionales que comunican ofertas y descuentos, buscando afectar las decisiones de com-pra de los consumidores y así estimular las ventas. Debido a ello, el proceso de percepción de precios del consumidor se realiza a través de la comparación del precio de referencia externo con los precios almacenados en la mente del consumidor, conocido como el precio interno; en ese sentido, las promociones en los precios provocan una reacción en la percepción del precio a pagar, tal como lo mencionan Kalwani y Yim (1992). "Las promociones actúan en la percepción del consumidor a través de distintas vías que actúan en un mismo sentido negativo (...) el primer lugar, un descuento en precios es una disminución comunicada del precio de (referencia externo), que el consumidor utiliza para comparar con la información de precios recordada (...) en consecuencia una disonancia entre el precio de referencia externo e interno provoca de inmediato una disminución de la percepción del precio (...) y en segundo lugar, un precio de oferta disminuye de manera contundente el precio de referencia interno".

Posterior al análisis de los datos, se lleva a cabo la comprobación de hipótesis, por lo tanto, se evidencia que las hipótesis relacionadas con el enmarcamiento ecológico y las estrategias de comunicación visual, no son comprobadas en el presente estudio, al no encontrarse relaciones mediante análisis factorial, lo que demuestra claramente que un producto ecológico al pretender comunicar únicamente que es amigable con el medio ambiente, o llevar colores asociados al código ecológico, no significa que inmediatamente los consumidores muestren un mayor grado de preferencia. Los resultados de esta investigación, demuestran que es necesario reorientar las estrategias de comunicación visual y con mayor incidencia para el posicionamiento de marcas verdes, pues lo que se viene logrando hasta el momento en consumo sustentable, supone un gran campo de acción para promover diversas estrategias que permitan que los productos amigables con el medio ambiente, generen mayor impacto visual en ciertos segmentos del mercado.

Hasta el momento la investigación de los códigos simbólicos culturales, basado en la teoría de las representaciones sociales de Abric (2001), es una técnica que ha hecho significativos aportes a la línea de investigación de procesos socioculturales, indicando que para el diseño y la comunicación visual en marcas verdes, existen elementos que contienen características fácilmente identificables por los consumidores como ecológicos tales como, el color, formas, figuras y tipografías. Facilitando así, el diseño de mensajes y conceptos publicitarios, necesarios para impactar en el consumidor.

No obstante, el resultado de la investigación, demuestra que al ubicar al comprador en una situación real de consumo, en donde se evalúa el precio, el tamaño, la presentación y los atributos, el consumidor suele comportarse inherente a las características de una marca verde, si esta no le garantiza que es ecológica, o por lo menos si no comunica, con elementos de alta calidad, que la compra garantiza el cuidado medioambiental, pues en muchos casos, los atributos ecológicos comunicados suelen formar parte de un truco publicitario.

En ese sentido, las marcas verdes deben buscar un nuevo posicionamiento que las identifique realmente, es por ello, que el sello ambiental colombiano juega un papel determinante en la diferenciación de los productos ecológicos, así como la posterior preferencia del consumidor, lo que implica orientar la investigación hacia los 
símbolos y elementos que resulten una verdadera creación de valor en los segmentos de mercado que prefieren una marca verde por sus características ambientales. Tal como lo menciona Fraj y Martínez (2003), en su estudio sobre el perfil psicográfico, el segmento de mercado que está dispuesto a pagar más por lo productos ecológicos, cuyos resultados obtenidos demuestran que existe una relación entre los valores y el comportamiento ecológico y los consumidores que siguen las últimas tendencias en modas, este tipo de segmentas de consumidores indican una mayor predisposición a ser más ecológicos y a pagar más por la adquisión de productos que cumplan con una promesa de valor medioambiental.

Por lo anterior, es preciso orientar estratégicamente la investigación del consumidor hacia el conocimiento de este mercado, y así, potencializar la oferta de este tipo de bienes y servicios mediante una implementación de las acciones estratégicas del Marketing fundamentadas en un escenario de consumo sostenible, en donde se comprenda ampliamente los procesos de decisión y compra de los consumidores y los diferentes procesos involucrados en su actitud frente a las marcas verdes y cómo desde las actividades de mercadeo, se logrará fortalecer un consumo ambientalmente responsable.

Finalmente, se considera relevante para el fortalecimiento de la línea de investigación en procesos socioculturales, el estudio acerca de cómo incrementar el nivel de motivación medioambiental de cada individuo dado el interés que muestran los individuos en querer tomar actitudes más ecológicas y en la compra de productos respetuosos con el medio ambiente. Desde luego, este tipo de información se puede construir optimizando el componente investigativo, desde la academia que permita generar un sistema informativo para el sector empresarial, como elemento que potencie la generación de una cultura de sustentabilidad. 


\section{REFERENCIAS BIBLIOGRÁFICAS}

AAaker, D. A. y Joachimsthaler, E. (2000). Brand Leadership. New York: The Free Press.

Abric, J., (2001) Prácticas sociales y representaciones sociales. México, Ediciones Coyoacán.

Ajzen, I. y Fishbein, M. (1974). Factors influencing intentions and the intention behavior relation. Human Relations. (27), 1-15.

Ajzen, I. y Fishbein, M. (1980). Understanding attitudes and predicting social behavior. Estados Unidos de Norteamérica: Prentice Hall.

Baños, A. (2001). Los secretos de los precios: De los dos lados del mostrador. Buenos Aires: Granica.

Briñol, P, Corte, L. y Becerra, A. (2001) Qué Es Persuasión. Biblioteca nueva. Madrid.

Calderón, S. (2013) Las Estrategias de Comunicación Visual basadas en Simbología Cultural. Tesis de Grado. Fundación Universitaria Konrad Lorenz. Bogotá.

Castellanos, M. (2009). El desarrollo sustentable y la globalización: lo que la lógica de mercado no contó. La Chronique des Amériques. Recuperado http://www.ieim.uqam.ca/IMG/pdf/Maria_Lorena.pdf.

Castro, S. y Rosemberg, A. (2000). Precios psicológicos. análisis de la percepción del consumidor. Revista electrónica Quipukamayoc. Pp 83-92. Recuperado http://sisbib.unmsm.edu.pe/bibvirtual/publicaciones/quipukamayoc/ 2000/primer/precios_psico.htm.

Coulter, Keith S., y Robin A. Coulter. (2007) Distortion of Price Discount Perceptions: the Right Digit Effect," Journal of Consumer Research, 34 (2), pp. 162-175.

Fraj, E. y Martínez, E. (2003). Análisis psicográfico del segmento de consumidores que está dispuesto a pagar más por los productos ecológicos. Esic Market.

Hartmann, P. Apaolaza, V. y Forcada, J. (2002) La influencia del posicionamiento Verde en la actitud hacia la marca. Universidad del país Vasco. Recuperado http://www.epum2004.ua.es/aceptados/206.pdf

Jacob, J. y Olson, J.C. (1977). Consumer response to Price: an attitudinal, information processing perspective. Recuperado http://books.google.com.co/books?hl=es\&lr=\&id=NgYr-TZ_9HMC\&oi=fnd\&pg=PA13\&dq=Jacob +J.+y+Olson,+J.C.+\%281977\%29.+Consumer+response+to+Price:+an+attitudinal,+information+processing +perspective.\&ots=OIW9Is48IS\&sig=yBCxH9J-rQ-proFG9UzvnPksGjw\#v=onepage\&q\&f=false

Kals, E., Schumacher, D., y Montada, L. (1999). "Emotional affinity toward nature as a motivational basis to protect nature. Environment and Behavior. 31, (2), 178-202. http://es.scribd.com/doc/143809056/Emotional-AffinityToward-Nature-as-a-Motivational-Basis-to-Protect-Nature. 
Kalwani, M., \& Yim, C. (1992). Consumer Price and Promotion Expectations: An Experimental Study. Journal of Marketing Research, pp. 29, 90-100.

Lichtenstein, D. Ridgway, N y Netemeyer, R. (1993) "Price Perceptions and Consumer Shopping Behavior: A Field Study. Recuperado de: http://www.jstor.org/discover/10.2307/3172830?uid=3737808\&uid=2129\&uid=2\&uid $=70 \&$ uid $=4 \&$ sid $=21102375879857$.

Monroe, K. (1973): "Buyers' Subjetive Perceptions of Price". Journal of Marketing Research. 10. 70-80.

Moscovici, S. (1976). Social influence and social change. London: Academic Press.

Oubiuña, J. (1997) "La Percepción de Precios de los Consumidores. Implicaciones para la Fijación de Precios Minoristas". Distribución y Consumo, (33). 88-100.

Ritov, I., \& Kahneman, D. (1997). How people value the environment: Attitudes versus economic values. In M.H. Bazerman, D.M. Messick, A.E. Tenbrunsel \& K.A. Wade-Benzoni, (Eds.), Environment, ethics, and behavior: The psychology of environmental valuation and degradation, pp. 33-51. San Francisco, CA: The New Lexington Press.

Sánchez, M., Grande, I., Gil, J., Gracia, A. (2001). Diferencias entre los segmentos del mercado en la disposición a pagar por un alimento ecológico: valoración contingente y análisis conjunto. Estudios Agrosociales y Pesqueros. (190), pp.141-163.

Terrón, E. (2009). Educación ambiental representaciones sociales de los profesores de educación básica y sus implicaciones educativas. Recuperado http://www.comie.org.mx/congreso/memoriaelectronica/v10/pdf/area_ tematica_03/ponencias/0983-F.pdf.

Thomas, M., \& Morwitz, V. (2005). Penny Wise and Pound Foolish: The Left Digit Effect in Price Cognition, Journal of Consumer Research, 22 (June), pp.54-64.

Velandia, S. (2012). Manifestaciones semióticas en la comunicación ecológica. Universidad Sergio Arboleda Recuperado http://www.usergioarboleda.edu.co/investigacion-comunicacion/documentos-comunicacion-4/semioticacomunicacion-ecologica.pdf. 


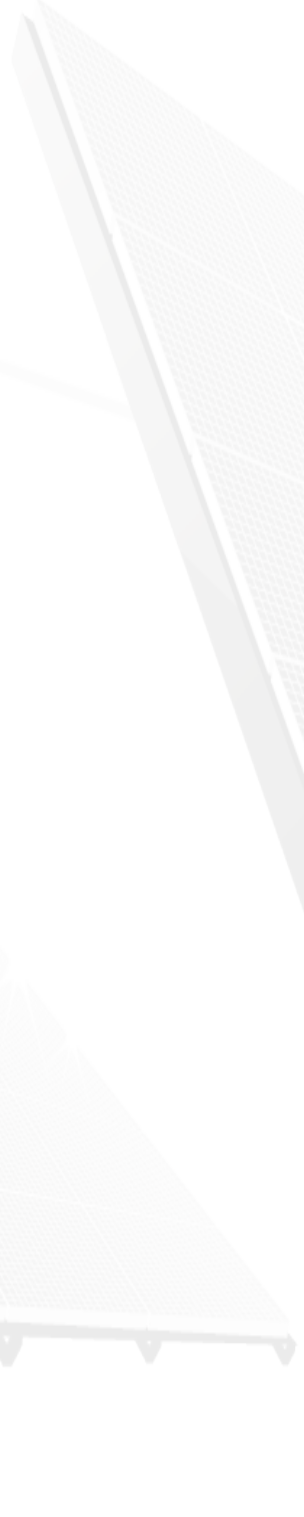

